\title{
On the unstable stacking criterion for ideal and cracked copper crystals
}

\author{
By Y. W. ZHANG, T. C. WANG and Q. H. TANG \\ LNM, Institute of Mechanics, Chinese Academy of Sciences, \\ Beijing 100080, PR China
}

[Received 19 September 1994† and accepted 3. March 1995]

\begin{abstract}
The unstable stacking criteria for an ideal copper crystal under homogeneous shearing and for a cracked copper crystal under pure mode II loading are analysed. For the ideal crystal under homogeneous shearing, the unstable stacking energy $\gamma_{\text {us }}$ defined by Rice in 1992 results from shear with no relaxation in the direction normal to the slip plane. For the relaxed shear configuration, the critical condition for unstable stacking does not correspond to the relative displacement $\Delta=b_{\mathrm{p}} / 2$, where $b_{\mathrm{p}}$ is the Burgers vector magnitude of the Shockley partial dislocation, but to the maximum shear stress. Based on this result, the unstable stacking energy $\Gamma_{\text {us }}$ is defined for the relaxed lattice. For the cracked crystal under pure mode II loading, the dislocation configuration corresponding to $\Delta=b_{\mathrm{p}} / 2$ is a stable state and no instability occurs during the process of dislocation nucleation. The instability takes place at approximately $\Delta=3 b_{\mathrm{p}} / 4$. An unstable stacking energy $\Pi_{\text {us }}$ is defined which corresponds to the unstable stacking state at which the dislocation emission takes place. A molecular dynamics method is applied to study this in an atomistic model and the results verify the analysis above.
\end{abstract}

\section{$\S 1$. INTRODUCTION}

In the past few years, much effort has been made to study crack tip processes so as to establish the transition criterion from ductile to brittle fracture. Rice and Thomson (1974) first realized that the competition of dislocation nucleation and cleavage at the crack tip is an important factor in the transition criterion.

Recently, Schoeck (1991), Rice (1992), Rice, Beltz and Sun (1992), Beltz and Rice (1991) and Wang (1995) reanalysed the nucleation and emission of dislocations from a stressed crack tip based on the Peierls concept. In Rice's work, in order to overcome the drawbacks of the Rice-Thomson approach which makes use of an elastic solution for a fully formed dislocation and introduces a poorly defined core cut-off, Rice introduced the unstable stacking-fault energy $\gamma_{\text {us }}$ as a parameter to characterize the resistance to dislocation nucleation. He also applied it to dislocation nucleation from a crack tip.

The unstable stacking-fault energy can be estimated by different methods. The Frenkel sinusoid was used by Rice (1992) and a quantum-mechanical method was applied by Paxton, Gumbsch and Methfessel (1991). Cheung, Yip and Argon (1991) used the embedded-atom method to calculate $\gamma_{\text {us }}$ for b.c.c. Fe and Zhou and Carlsson (1993) used the Green function method to evaluate $\gamma_{\text {us }}$ for an h.c.p. crystal. However,

$†$ Received in final form 24 February 1995. 
the estimates show differences for different methods and so there is some uncertainty in $\gamma_{\text {us. }}$.

In the present paper, for the configuration with no relaxation in the direction normal to the slip plane, a computational cell in which all atoms undergo a pure shear displacement is used to calculate the relation between the relative displacement $\Delta$ and shear stress $\tau$ by using a Finnis-Sinclair potential. The relation of $\Delta$ and $\tau$ is approximately a sinusoidal function. For a cell in which relaxation in the direction normal to the slip plane is allowed, its instability is analysed by a molecular dynamics method. We find that the instability occurs at the maximum shear stress. Based on this concept, the unstable stacking-fault energy $\Gamma_{\text {us }}$ for the ideal relaxed crystal is defined. Our analysis shows that, owing to the atomic characteristics and inhomogeneous shear at the crack tip, the Peierls concept is not applicable. The molecular dynamics method is also used to calculate the nucleation and emission of a partial dislocation from a crack tip. The analysis shows that the fully formed dislocation, that is $\Delta=b_{\mathrm{p}} / 2$, does not correspond to the stacking instability. (Here, $b_{\mathrm{p}}$ is the magnitude of the Burgers vector of the Shockley partial.) The instability takes place approximately at $\Delta=3 b_{\mathrm{p}} / 4$, which just corresponds to the emission of a dislocation from the crack tip. Based on this analysis, the unstable stacking energy $\Pi_{\mathrm{us}}$, for dislocation emission from a crack tip is defined.

\section{§ 2. LATTICE WITH UNRELAXED HOMOGENEOUS SHEAR}

By using the Peierls (1940) concept, Rice (1992) assumed that the shear stress $\tau$ along the slip plane is a periodic function of slip displacement $\Delta$. The relation of $\tau$ and $\Delta$ is assumed to have a form like that in fig. 1 . The unstable stacking fault energy defined by Rice is also illustrated in fig. 1 . If the relation of $\tau$ and $\Delta$ is taken to be a sinusoidal function, that is

$$
\tau=\tau_{\mathrm{m}} \sin \left(\frac{2 \pi \Delta}{b}\right)
$$

the energy per unit area of the slip plane is

$$
\Psi=\int \tau \mathrm{d} \Delta
$$

Fig. 1

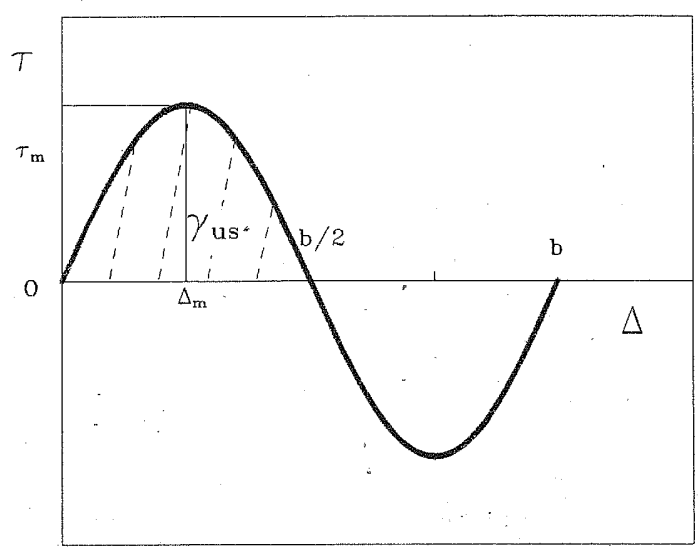

Illustration of the periodic relation between shear stress and relative shear displacement and the unstable stacking energy $\gamma_{u s}$ defined by Rice. 
and $\gamma_{\mathrm{us}}$ is the maximum value of $\Psi$ for the sinusoidal function, that is

$$
\gamma_{\mathrm{us}}=\frac{\tau_{\mathrm{m}} b}{\pi}
$$

In the present paper, the relative shearing of elastic bodies assumed by Peierls is simulated by an atomistic method, that is all atoms move through the prescribed homogeneous shear displacement. The interatomic potential used here is the ' $N$-body' potential of the form proposed by Finnis and Sinclair (1984) and constructed by Ackland, Tichy, Vitek and Finnis (1987). The atomic level stress $\sigma_{i}^{\alpha \beta}$ associated with atom $i$ is calculated by using the formula of Alber et al. (1992).

The $\{110\},\{111\}$ and $\{112\}$ crystallographic planes bound the parallelepiped with a periodic boundary condition used along the $\langle 112\rangle$ and $\langle 110\rangle$ directions. The cases of (a) shearing along the $\langle 112\rangle$ direction, which corresponds to the displacement direction of a partial dislocation, and $(b)$ shearing along the $\langle 110\rangle$ direction, which corresponds to the displacement direction of a perfect dislocation, are considered. The calculated relations of the relative displacement $\Delta$ against the shear stress $\tau$ for cases $(a)$ and $(b)$ are shown in figs. $2(a)$ and $(b)$ respectively. The relation of case $(a)$ shows an

Fig. 2

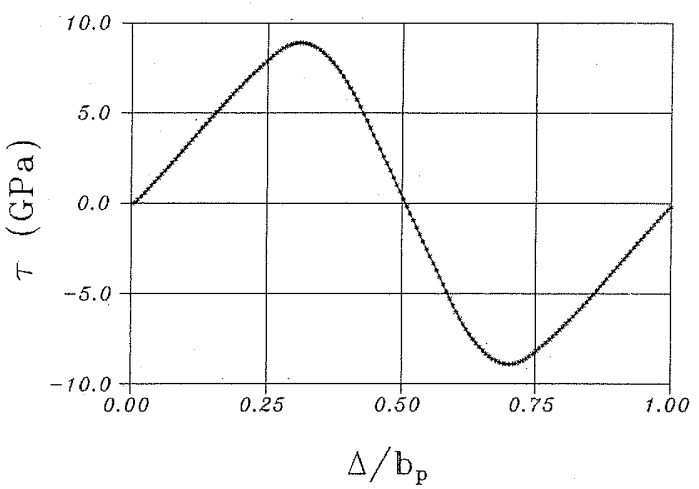

(a)

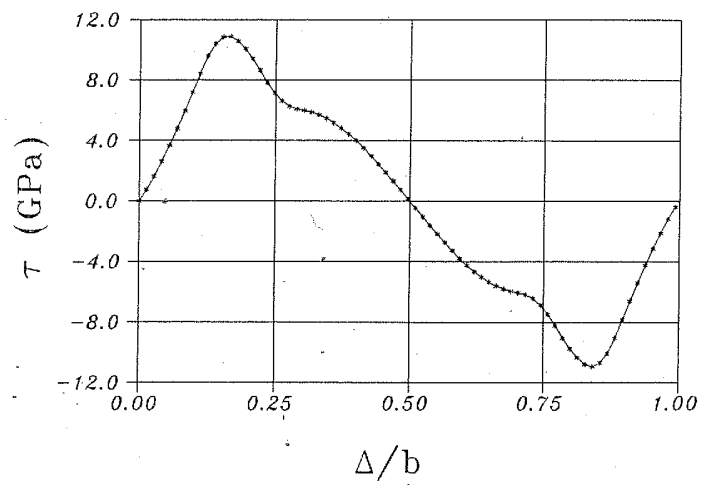

(b)

The relative shear displacement against shear stress under the unrelaxed shear: $(a)$ shear, $\langle 112\rangle\{111\} ;(b)$ shear, $\langle 110\rangle\{111\}$. 
approximately sinusoidal function, but that of case $(b)$ is different. The occurrence of lattice instability under the unrelaxed shearing, which just corresponds to the formation of a dislocation, takes place at $\Delta=b / 2$, where $b$ is the magnitude of $\frac{1}{2}\langle 110\rangle$, for the perfect dislocation and at $\Delta=b_{\mathrm{p}} / 2$ for the partial dislocation. The maximum shear stress roughly corresponds to the theoretical shear strength of copper. The unstable stacking fault energy $\gamma_{\mathrm{us}}$ defined by Rice is $0.381 \mathrm{~J} \mathrm{~m}^{-2}$ for case $(a)$ and $0.409 \mathrm{~J} \mathrm{~m}^{-2}$ for case (b). The value for $(a)$ is a little larger than that obtained by Rice (1992) using the Frenkel relation.

\section{§3. LATTICE WITH RELAXED HOMOGENEOUS SHEAR}

In the preceding analysis, all the atoms were constrained by prescribing the shear displacement. Now we consider instability of a lattice when relaxation of atoms in the direction normal to the slip plane is allowed.

In the same model as above, the discrete border atoms were displaced by the homogeneous shearing displacement and the inner region atoms relax by Newton's law using the leapfrog algorithm. Blocks with either 12 or $15\{111\}$ planes were used. The top five and bottom five planes were rigidly displaced by the homogeneous shear displacement; so the free atoms were only in either two or five $\{111\}$ planes, as indicated by the full circles in figs. $3(\dot{a})$ and $(b)$ respectively. For shearing along the $\langle 112\rangle$, atom motion parallel to the $\langle 110\rangle$ direction is prohibited.

The curves of the relative shear displacement $\Delta$ against shear stress $\tau$ for the different pairs of planes shown in figs. $3(a)$ and $(b)$ are given in figs. $4(a)$ and $(b)$. From fig. $4(a)$ it can be seen that the pair of planes 2 shown in fig. $3(a)$ is displaced by a partial Burgers vector, but at the same time unloading takes place at the pairs 1 and 3 .

Fig. 3

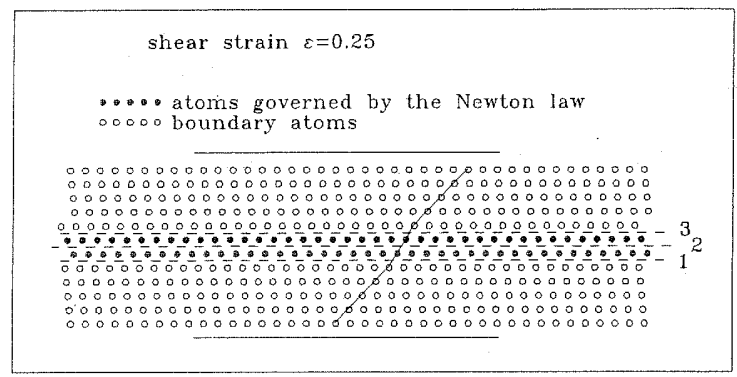

(a)

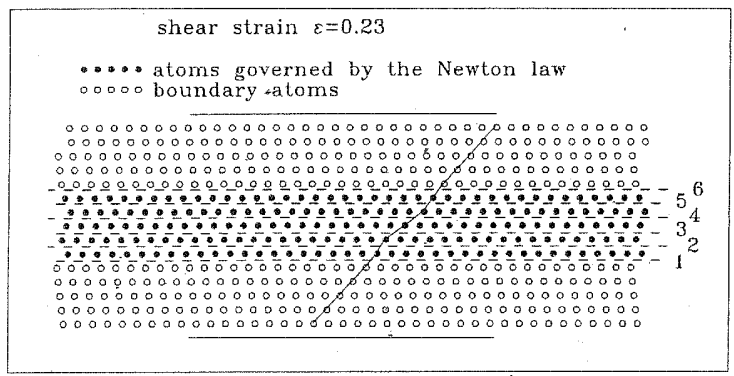

(b)

The simulated atom configurations under the relaxed shear with $(a)$ two relaxed planes and $(b)$ five relaxed planes. 
Fig. 4

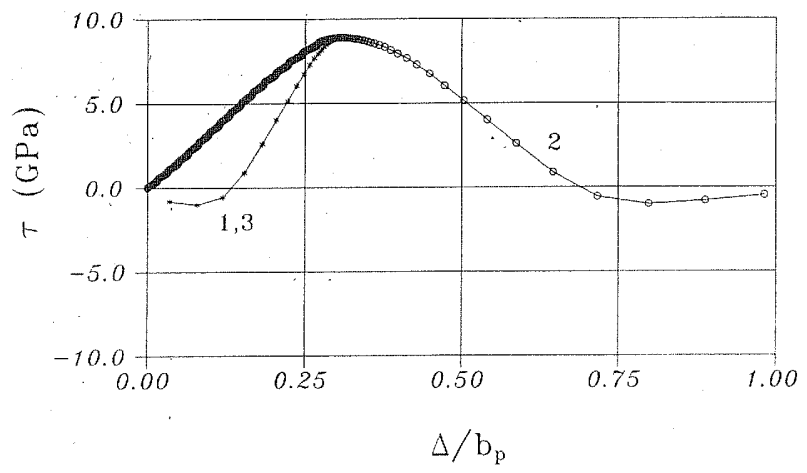

(a)

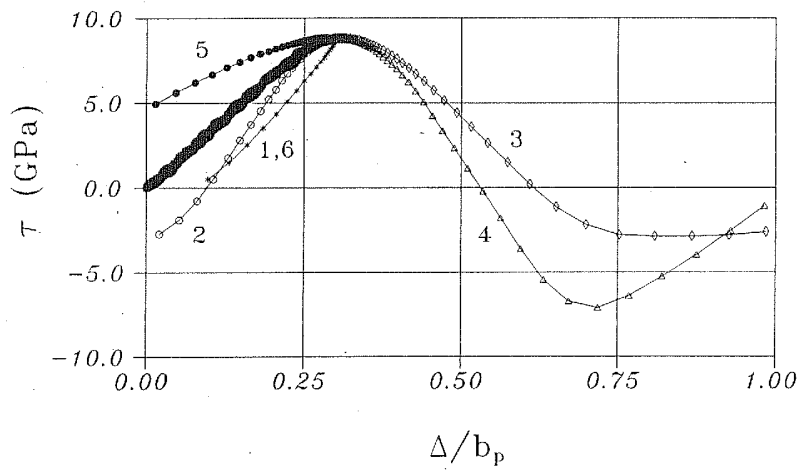

(b)

The relative shear displacement against shear stress for the different pairs of atom planes under the relaxed shear for $(a)$ two relaxed planes and $(b)$ five relaxed planes.

From fig. $4(\mathrm{~b})$ it can be seen that the pairs 3 and 4 shown in fig. $3(b)$ are displaced by a partial Burgers vector, while unloading takes place at the pairs 1 and 2 and pairs 5 and 6. For cases with more $\{111\}$ planes, a similar phenomenon is observed.

From this analysis, an instability takes place at the maximum shear stress $\tau_{\mathrm{m}}$. Based on this critical condition, a new unstable stacking-fault energy for the relaxed lattice under homogeneous shearing is defined:

$$
\Gamma_{\mathrm{us}}=\int_{0}^{\Delta_{\mathrm{m}}} \tau \mathrm{d} \Delta,
$$

where $\Delta_{\mathrm{m}}$ is the relative shear displacement corresponding to the maximum shear stress.

\section{§4. INSTABILITY OF THE LATTICE AT A CRACK TIP}

The results of the homogeneous shearing were applied locally to the states of inhomogeneous shear by Rice (1992). At a crack tip, the stress is very inhomogeneous; so it is essential to treat the effect of this on instability of the lattice at the tip. Owing to the nonlinear and many-body nature, a rigorous mathematical analysis for lattice instability is not possible; so now we consider the implication of the method used in the present paper. 
Fig. 5

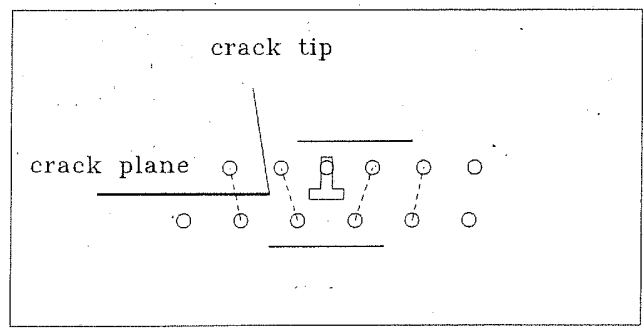

(a)

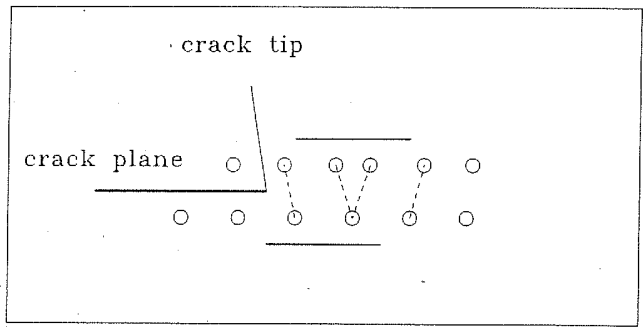

(b)

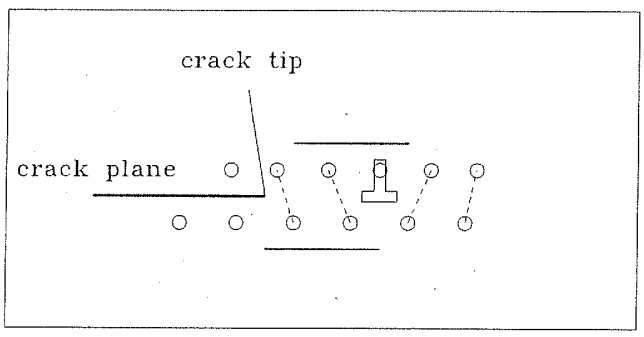

(c)

(a) The atomic configuration for the formation of a partial dislocation; $(b)$ the critical unstable state of this partial dislocation; and $(c)$ the atomic configuration for the movement by a partial Burgers vector from the crack tip.

\subsection{Analysis}

A dislocation is a metastable configuration in a crystal and a virtual force must be applied to the dislocation to make it move. Under the mode II loading, an incipient partial dislocation gradually grows into a fully formed dislocation, that is $\Delta=b_{\mathrm{p}} / 2$. If one wants to make the dislocation move further, a force must be applied; so the unstable configuration does not correspond to dislocation formation, that is $\Delta=b_{\mathrm{p}} / 2$, but to dislocation emission from the crack tip. Figure $5(a)$ shows a fully formed dislocation in a s.c. crystal, which is a metastable state, fig. $5(b)$ corresponds to the critical unstable state and fig. $5(\mathrm{c})$ represents the movement of the dislocation by a partial Burgers vector. Hence the instability corresponds to the emission of a dislocation from a crack tip.

For the crack tip processes, $\gamma_{\mathrm{us}}$ defined by Rice corresponds to the energy required for the formation of a dislocation, not to the instability of dislocation emission. A solid state parameter $\Pi_{\mathrm{us}}$, the unstable stacking energy for dislocation emission from the crack tip, can be defined: 


$$
\Pi_{\mathrm{us}}=\int_{0}^{\Delta_{\mathrm{us}}} \tau \mathrm{d} \Delta
$$

where $\Delta_{\text {us }}$ is the relative shear displacement at which the nucleated dislocation at the crack tip becomes unstable. Thus the critical condition for the emission of a dislocation is

$$
J=\Pi_{\mathrm{us}}
$$

where $J$ is the $J$ integral.

\subsection{Verification}

We consider the state of an initial rectangular lattice with a slit under pure mode II loading. The molecular dynamics method is employed to simulate the crack tip processes.

A parallelepiped block in the same orientation as before, but with a slit, was used. The crack front is along $\langle 110\rangle$ and the crack grows along $\langle 112\rangle$ direction, so that the dislocation nucleated from crack tip is a Shockley partial. In order to ensure the traction-free condition on the crack planes, the atom interaction across the crack planes must be prohibited. To do this, the model is divided into cells, each with a size a little larger than the cut-off distance of the potential. Atoms in a cell adjacent to the crack plane are not allowed to interact with the atoms in cells across the crack plane. The 'fixed-boundary' method is used, that is the position of border atoms is prescribed by the pure mode II $K$ displacement field. Although this method is less accurate than the 'flexible-boundary' method, since we only investigate the dislocation nucleation process far from the border, the difference between the two methods is therefore small (deCelis, Argon and Yip 1983). Relaxed atomic configurations were examined for increasing applied displacement.

The atomic configuration corresponding to $K_{\mathrm{II}}=0.26 \mathrm{MPa} \mathrm{m}^{1 / 2}$ is shown in fig. 6 . An emitted partial dislocation can be clearly observed. The variation in the relative shear displacement against the shear stress for the pair of atoms at the crack tip is shown in fig. 7. It can be seen that the instability does not occur at $\Delta=b_{\mathrm{p}} / 2$. To make the dislocation move away from the crack tip, a shear stress must be applied and the instability occurs approximately at $\Delta=3 b_{\mathrm{p}} / 4$. It can also be seen that the formation of a dislocation requires a large shear stress and energy. If a dislocation has been fully formed, it requires a relatively small shear stress and energy to drive it away from the crack tip. From fig. 7 , it can be seen that $\Pi_{\mathrm{us}}$ is actually equal to $\gamma_{\text {us }}$ defined by Rice plus the energy for driving the dislocation to the unstable state of emission $\gamma_{\text {dus }}$, that is

$$
\Pi_{\mathrm{us}}=\gamma_{\mathrm{us}}+\gamma_{\mathrm{dus}}
$$

Compared with $\gamma_{\mathrm{us}}, \gamma_{\mathrm{dus}}$ is relatively small and $\gamma_{\mathrm{us}}$ is the main part of $\Pi_{\mathrm{us}}$.

Our results also show that critical stress intensity factor for the dislocation nucleation $K_{\mathrm{II}}^{\mathrm{n}}$ is $0.21 \mathrm{MPa} \mathrm{m}^{1 / 2}$ in our model of copper. The $\gamma_{\mathrm{us}}$ directly obtained from the crack tip is equal to $0.221 \mathrm{~J} \mathrm{~m}^{-2}$, which is in good agreement with $0.22 \mathrm{~J} \mathrm{~m}^{-2}$ obtained by Rice (1992). The calculated $\Pi_{\text {us }}$ is $0.26 \mathrm{~J} \mathrm{~m}^{-2}$. The stress intensity factor for dislocation emission from the crack tip is $K_{\mathrm{II}}^{\mathrm{e}}=0.22 \mathrm{MPa} \mathrm{m}^{1 / 2}$, which is very close to $K_{\text {II }}^{\mathrm{n}}$, implying that, once a dislocation has been formed, it will be emitted from the crack tip very easily. 
Fig. 6

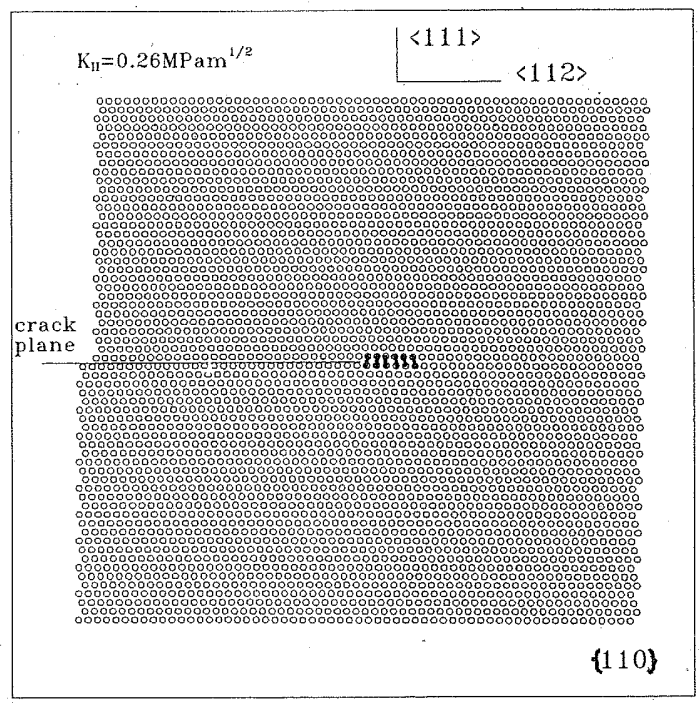

The stressed atomic configuration at $K_{\Pi}=0.26 \mathrm{MPam}^{1 / 2}$, at which a partial dislocation is emitted.

Fig. 7

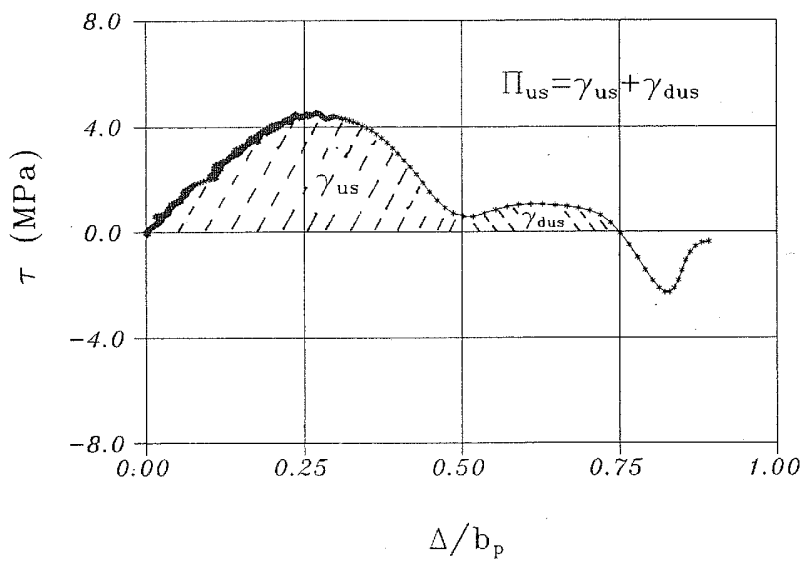

The relative shear displacement against shear stress for a pair of atoms at the crack tip, and the illustration of $\gamma_{\mathrm{us}}$ defined by Rice and $\gamma_{\text {dus }}$ and $\Pi_{\mathrm{us}}$ defined in the present paper.

\section{$\S 5$. CONCLUSIONS}

For an ideal copper crystal under homogeneous shearing, the unstable stacking-fault energy $\gamma_{u s}$ defined by Rice (1992) is for the unrelaxed configuration. The relations between the relative shear displacement and shear stress for both the partial and the perfect dislocation displacements of the unrelaxed lattice and $\gamma_{\text {us }}$ have been obtained. For the relaxed configuration, the critical condition of unstable stacking does not correspond to the relative displacement $\Delta=b_{\mathrm{p}} / 2$ but to shear stress $\tau=\tau_{\mathrm{m}}$. Based on this concept, the unstable stacking-fault energy $\Gamma_{\text {us }}$ is defined. For a cracked crystal under pure mode II loading, the incipient dislocation configuration corresponding to $\Delta=b_{\mathrm{p}} / 2$ is a stable state, no instability occurs during this process and $\gamma_{\mathrm{us}}$ is just 
the energy required for the formation of a dislocation. Instability takes place after the formation of the dislocation at approximately $\Delta=3 b_{\mathrm{p}} / 4$, which just corresponds to the emission of the dislocation. A new unstable stacking-fault energy $\Pi_{\mathrm{us}}$ is defined which corresponds to this unstable state. The results of a molecular dynamics study verify this analysis. However, the extra energy $\gamma_{\text {dus }}$ is much smaller than $\gamma_{\text {us }}$ and $\Pi_{\text {us }}$ is approximately equal to $\gamma_{\mathrm{us}}$. Since both $\Pi_{\mathrm{us}}$ and $\gamma_{\mathrm{us}}$ are much smaller than the energy required for cleavage, the qualitative model of Rice for the ductile-to-brittle transition is unaffected by the present results.

\section{ACKNOWLEDGMENTS}

The project is supported by the National Natural Science Foundation of China. The authors are grateful to Professor Zhou Fuxin for helpful discussion.

\section{REFERENCES}

ACKLAND, G. J., Tichy, G., Vitek, V., and Finnis, M. W., 1987, Phil. Mag. A, 56, 735.

Alber, I., Bassani, J. L., Khantha, M., Vitek, V., and Wang, G. J., 1992, Phil. Trans. R. Soc., $339,555$.

BELTZ, G. E., and RICE, J. R., 1991, Modelling the Deformation of Crystalline Solids, edited by T. C. Lowe, A. D. Rollett, P. S. Follansbee and G. S. Daehn (Warrendale, PA: Minerals, Metals and Materials Society), p. 457.

Chieung, K., YIP, S., and Argon, A. S., 1991, J. appl. Phys., 69, 2088.

DECELis, B., Argon, A. S., and YIP, S., 1983, J. appl. Phys., 54, 4864.

Finnis, M. W., and SinClair, J. E., 1984, Phil. Mag., 50, 45.

Paxton, A. T., Gumbsch, P., and Methfessel, M., 1991, Phil. Mag. Lett., 63, 267.

Pejerls, R. E., 1940, Proc. phys. Soc., 35, 379.

Rice, J. R., 1992, J. Mech. Phys. Solids, 40, 239.

RICE, J.R., BeLTZ, G. E., and Sun, Y., 1992, Topics in Fracture and Fatigue, edited by A. S. Argon (Berlin: Springer), p. 1.

RiCe, J. R., and ThOMSOn, R., 1974, Phil. Mag., 29, 73.

SCHOECK, G., 1991, Phil. Mag., 40, 111.

WANG, T. C., 1995, Int. J. Fract. (in press).

Zhou, S. J., and Carlsson, A. E., 1993, Phys. Rev. B, 47, 7710. 